\title{
Numerical Analysis on Space Charge and AC-DC Combined Breakdown Strength in Polyethylene
}

\author{
Siyu Zhang and Zongren Peng \\ State Key Laboratory of Electrical Insulation and Power Equipment \\ Xi'an Jiaotong University, \\ Xi'an 710049, Shaanxi, P. R. China \\ Churui Zhou and George Chen \\ University of Southampton \\ School of Electronics and Computer Science \\ Southampton, UK
}

\begin{abstract}
In high voltage direct current (HVDC) power transmission systems, electrical equipment at the valve side usually withstands complex stresses like AC-DC combined voltages. Understanding the charge migration and accumulation characteristics under complex stresses and their influences on the breakdown strength are of great significance. This paper presents an in-depth simulation study on the AC-DC combined breakdown strength using a modified bipolar charge transport model, which takes the deterioration of materials into consideration. Firstly, the simulation results of charge profiles under $\mathrm{AC}$ voltages agree well with experimental results, which partly illustrates the appropriate selection of parameters. Then the AC breakdown strengths with different ramping rates and frequencies are studied. Charges accumulated relatively close to the surface of samples can cause severe distortion of the electric field after the change of voltage polarity. After that, the breakdown voltages with different ratios of the AC to DC component are calculated and analyzed, especially on how AC and DC component influence the charge migration and accumulation during the evolution of the aging process eventually leading to breakdown. The simulation results of AC-DC combined breakdown voltages show good agreement with previous experimental results.
\end{abstract}

Index Terms - space charge, simulation, dielectric breakdown, charge transport

\section{INTRODUCTION}

HIGH voltage direct current (HVDC) systems can transmit power over longer distances with higher stability, lower cost and lower loss. These outstanding advantages over high voltage alternating current (HVAC) systems have made HVDC a better choice for power transmission over long distances. However, in converter stations and valve halls, a large part of electrical equipment suffers from harmonics, which are superimposed AC upon DC voltages. Whether insulation materials can maintain the dielectric strength under complex stresses is of great significance to the safe operation of HVDC systems.

Many researchers have studied the breakdown strengths of insulation materials under composite $\mathrm{AC}$ and $\mathrm{DC}$ voltages through experiments. The breakdown strength of oil barely changes with $\mathrm{AC}$ ratio while that of oil-impregnated paper decreases with increasing $\mathrm{AC}$ ratio [1,2]. For cross-linked polyethylene, the breakdown voltage firstly shows a slight increase followed by a rapid decrease [3, 4]. However, the underlying mechanism of how the AC component influences the breakdown process in polyethylene remains unknown.

Manuscript received on 23 August 2018, in final form 18 November 2018, accepted xx Month 20yy. Corresponding author: S. Zhang.
The role of space charge accumulation in material degradation under DC voltages has been thoroughly studied, which includes distortion of electric field, acceleration of tree growth and eventually failure of insulation [5, 6]. However, space charge characteristics under AC and AC-DC combined voltages are less studied through experiments due to the difficulty in data recording and processing for charge profiles at different phase angles. Without comparison with experimental results, the selection of parameters for simulation study on either AC or AC-DC combined breakdown strengths lacks credibility. Based on our previous work on AC space charge measurements, charge accumulation near the surface of polyethylene can cause severe field distortion after the $\mathrm{AC}$ voltage polarity change [7]. However, the role of trapped space charges under AC voltages in the degradation of materials and breakdown process lacks comprehensive understanding. As an extended study, this paper mainly focuses on simulating the evolution of aging process leading to breakdown processes under AC and AC-DC combined voltages.

In this paper, the modified bipolar charge transport model, which takes the degradation of the dielectric into account, is firstly introduced, followed by a detailed explanation on how the physical parameters of charge transport and trapping 
process would change with aging. Secondly, the charge evolution in polyethylene under AC voltage is simulated. The good agreement between simulation and experimental results verifies that the selected parameters are applicable. Thirdly, the variation of breakdown strengths with frequency and ramping rate of $\mathrm{AC}$ voltage is calculated and analyzed from the perspective of charge accumulation. Then the relationship between the ratio of $\mathrm{AC}$ to $\mathrm{DC}$ component and AC-DC combined breakdown strength is studied. From the analysis, the amplitude and application duration of AC component will influence the injection and migration speed of charge carriers. The numerical relationship acquired through simulation is in accordance with that obtained from previous experiments, which validates the feasibility of the modified model.

\section{SIMULATION MODEL AND EXPERIMENTAL PLATFORM}

\subsection{BIPOLAR CHARGE TRANSPORT MODEL}

The bipolar charge transport model, proposed in 2006, has been widely used to investigate the relationship between charge transport and conduction, electrical breakdown and surface potential decay [8].

Charge dynamics inside dielectrics include charge injection, charge trapping, de-trapping and recombination processes, as shown in Figure 1. Here, a one-dimensional system with a thickness of $\mathrm{d}$ is considered. $x=0$ is connected to the ground electrode while $x=\mathrm{d}$ is connected to the high voltage electrode.

Charge carriers are provided by injection from electrodes, which follows the Schottky thermionic emission law:

$$
j_{e, h}(x, t)=A T^{2} \exp \left(-\frac{e w_{\text {ei,hi }}}{k T}\right) \exp \left(\frac{e}{K T} \sqrt{\frac{e E(x, t)}{4 \pi \varepsilon}}\right)
$$

where $j_{e}, j_{h}$ are the fluxes of electrons and holes in $\mathrm{A} / \mathrm{m}^{2}, A$ is the Richardson constant that equals to $1.2 \times 10^{6} \mathrm{~A} /\left(\mathrm{m}^{2} \cdot \mathrm{K}^{2}\right), T$ is temperature in $\mathrm{K}, e$ is elementary charge in $\mathrm{C}, w_{\mathrm{ei}}$ and $w_{\mathrm{hi}}$ are the injection barriers for electrons and holes in $\mathrm{eV}, k$ is the Boltzmann constant that equals to $1.38 \times 10^{-23} \mathrm{~m}^{2} \cdot \mathrm{kg} /\left(\mathrm{s}^{2} \cdot \mathrm{K}\right), E$ is electric field strength in $\mathrm{kV} / \mathrm{mm}, \varepsilon$ is the dielectric permittivity of the sample in $\mathrm{F} / \mathrm{m}$.

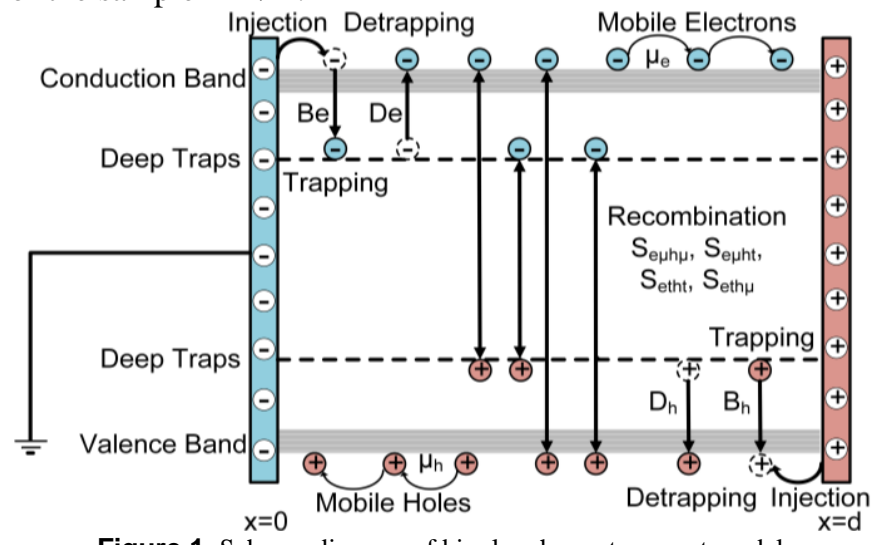

Figure 1. Scheme diagram of bipolar charge transport model.

Two types of traps exist in insulating materials like polyethylene: physical traps and chemical traps. Physical traps, usually with a depth smaller than $0.5 \mathrm{eV}$, are the conformational defects in the amorphous region. Chemical traps, with a depth ranging from 0.04 to $1.53 \mathrm{eV}$, are related to impurities and chemical defects existing at the interphase between crystalline region and amorphous region [9]. These traps interfere with the movement of charge carriers $[10,11]$. Charges in shallow traps could hop or tunnel to another shallow trap only after a short stay. However, it is extremely hard for charges in deep traps to escape and migrate again. Molecular simulation results show the residence time for a $0.1 \mathrm{eV}$ trap is only $10^{-13} \mathrm{~s}$ while that for a $1 \mathrm{eV}$ trap is 500s [12]. Thus, it is assumed that charges migrate through hopping in and out of shallow traps. If deep traps capture charges, these charges can no longer escape.

The mobility defined by hopping mechanism is:

$$
\mu_{e, h}(x, t)=\left(\frac{2 v_{T A H} a}{E(d, t)}\right) \exp \left(\frac{\Delta U_{e, h}}{k_{B} T}\right) \sinh \left(\frac{a e E(d, t)}{2 k_{B} T}\right)
$$

where $v_{\text {ATE }}$ is the escape frequency, $a$ is the distance between traps, $\Delta U_{\mathrm{e}}$ and $\Delta U_{\mathrm{h}}$ are the trap depths for electrons and holes.

The movement of charges follows the continuity equation, charge transport equation and Poisson's equation.

$$
\left\{\begin{array}{c}
\frac{\partial n(x, t)}{\partial t}+\frac{\partial j(x, t)}{\partial x}=s_{a}(x, t) \\
j(x, t)=\mu(x, t) n(x, t) E(x, t) \\
\frac{\partial E(x, t)}{\partial x}=\frac{\rho(x, t)}{\varepsilon}
\end{array}\right.
$$

where $\rho$ is the net charge density in $\mathrm{C} \cdot \mathrm{m}^{-3}, s_{\mathrm{a}}$ is the source term.

The extraction of electrons and holes at the electrodes are in the form:

$j_{e, h}(x, t)=n_{e \mu, h \mu}(x, t) \mu_{e . h}(x, t) E(x, t)$

The Langevin recombination model is used to define the recombination rates $[13,14]$ :

$S_{1}(x, t)=\mu_{e}(x, t) / \varepsilon_{0} \varepsilon_{r}$

$S_{2}(x, t)=\mu_{h}(x, t) / \varepsilon_{0} \varepsilon_{r}$

$S_{3}(x, t)=\left[\mu_{e}(x, t)+\mu_{h}(x, t)\right] / \varepsilon_{0} \varepsilon_{r}$

where $S_{1}$ is the recombination rate between mobile electrons and trapped holes, $S_{2}$ is the recombination rate between mobile holes and trapped electrons, $S_{3}$ is the recombination rate between mobile electrons and mobile holes.

An appropriate algorithm is very important to obtain an accurate solution without oscillations. The continuity equation is solved by using the SPLITTING method, which calculates the advection term and reaction term separately. A three-order QUICKEST scheme is used to solve the advection term [15]. A flux limiter is added to avoid oscillations and negative values [16]. A fourth-order Runge-Kutta method is used to calculate the reaction term. The simulation software is MATLAB.

\subsection{MATERIAL DEGRADATION EFFECT}

Recombination between positive and negative charge carriers in polyethylene can emit 4-5 eV energy, which is large enough to dissociate a molecule into radicals. The radicals then act as trap centers [10, 17]. During AC breakdown tests, energy generated through the frequent recombination can modify the structure inside polyethylene and lead to material degradation [18], which should be taken into consideration when simulating the charge evolution process under AC voltages.

With more energy emitted through recombination, more radicals are generated to function as traps. Thus, the trap density increases with the increasing number of recombined charges $[11,17]$. Since the trapping probability is closely related to trap density, the trapping coefficients also increase with 
recombination [19, 20]. Based on [21], the difference between the electron trap density of aged and unaged samples is assumed much larger than the difference between the hole trap density of aged and unaged samples. With the same aging time, the rate of increase of the trap density for electrons is much larger than that for holes. Furthermore, according to experimental results $[21,22]$, trap depths show an increase while injection barriers show an obvious decrease with the degradation of materials.

For simplification, the variation of physical parameters with the increasing number of recombined charges is set linear, as a preliminary simulation study to get qualitative results,

$d Y_{e, h} / d t=C_{e, h} \times\left(S_{1} n_{e \mu} n_{h t}+S_{2} n_{e t} n_{h \mu}+S_{3} n_{h \mu} n_{e \mu}\right)$

where $Y_{\mathrm{e}, \mathrm{h}}$ is the specific parameter, $C_{\mathrm{e}, \mathrm{h}}$ is the degradation rate for the specific parameter. The values of parameters for numerical simulation are shown in Table 1, which are based on [23] and experimental results.

Table 1. Parameters for simulation.

\begin{tabular}{|c|c|c|c|}
\hline \multirow{2}{*}{ Parameter } & \multicolumn{2}{|c|}{ Value } & \multirow{2}{*}{ Unit } \\
\hline & Electrons & Holes & \\
\hline Original injection barrier $w_{\mathrm{ei}} / w_{\mathrm{hi}}$ & 1.18 & 1.21 & $\mathrm{eV}$ \\
\hline Original Trap depth $\Delta U_{\mathrm{e}} / \Delta U_{\mathrm{h}}$ & 0.605 & 0.645 & $\mathrm{eV}$ \\
\hline $\begin{array}{l}\text { Original trapping coefficients } \\
\qquad B_{\mathrm{e}} / B_{\mathrm{h}} \\
\end{array}$ & 0.002 & 0.0005 & $\mathrm{~s}^{-1}$ \\
\hline Original trap density $N_{\text {te }} / N_{\text {th }}$ & 100 & 10 & $\mathrm{C} \cdot \mathrm{m}^{-3}$ \\
\hline $\begin{array}{l}\text { Degradation rate for trap density } \\
\qquad \gamma_{\mathrm{te}} / \gamma_{\mathrm{th}}\end{array}$ & 0.5 & 0.05 & \\
\hline $\begin{array}{c}\text { Degradation rate for trapping } \\
\text { coefficients } \beta_{\mathrm{e}} / \beta_{\mathrm{h}} \\
\end{array}$ & $1.67 \times 10^{-5}$ & $8.33 \times 10^{-6}$ & $\mathrm{~m}^{3} \cdot \mathrm{s}^{-1} \cdot \mathrm{C}^{-1}$ \\
\hline $\begin{array}{l}\text { Degradation rate for injection } \\
\text { barrier } \chi_{\mathrm{e}} / \chi_{\mathrm{h}}\end{array}$ & $-6.67 \times 10^{-6}$ & $-6.67 \times 10^{-6}$ & $\mathrm{eV} \cdot \mathrm{m}^{3} \cdot \mathrm{C}^{-1}$ \\
\hline $\begin{array}{l}\text { Degradation rate for trap depth } \\
\qquad \eta_{\mathrm{e}} / \eta_{\mathrm{h}}\end{array}$ & $3.33 \times 10^{-6}$ & $3.33 \times 10^{-6}$ & $\mathrm{eV} \cdot \mathrm{m}^{3} \cdot \mathrm{C}^{-1}$ \\
\hline Permittivity $\varepsilon$ & \multicolumn{2}{|c|}{$2.3 \times 8.85 \times 10^{-12}$} & $\mathrm{~F} \cdot \mathrm{m}^{-1}$ \\
\hline Temperature $T$ & \multicolumn{2}{|c|}{300} & $\mathrm{~K}$ \\
\hline
\end{tabular}

\subsection{AC SPACE CHARGE MEASUREMENT SYSTEM}

The schematic diagram of AC space charge measurement system is shown in Figure 2.

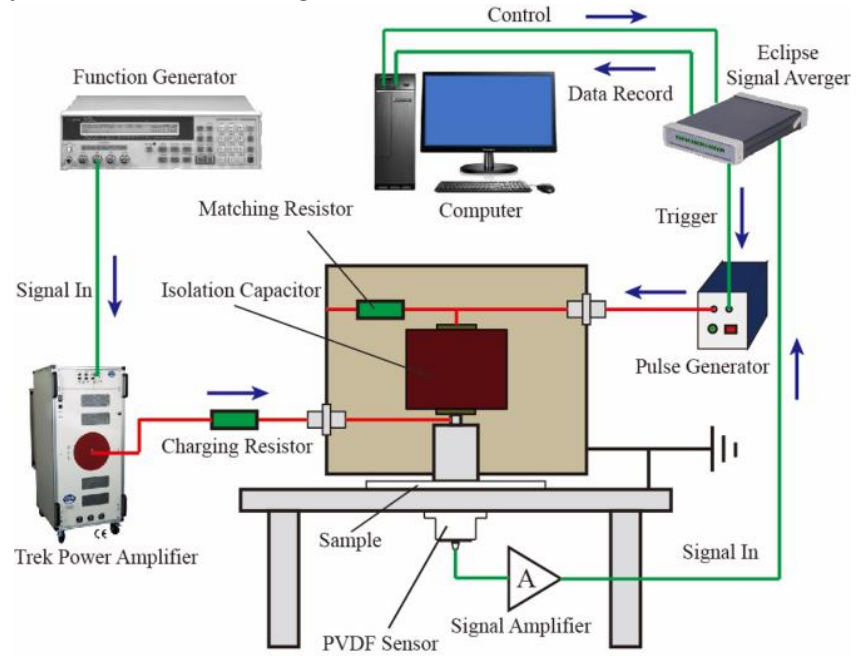

Figure 2. AC space charge measurement platform.

Samples are positioned between flat bottom and top electrode in the pulsed electro-acoustic (short as PEA) system. High voltage is applied to the PEA system by using a function generator and a TREK power amplifier. The highest repetitive frequency of pulse generator is $2 \mathrm{kHz}$. The pulse width is $5 \mathrm{~ns}$. The amplitude of pulse is $1 \mathrm{kV}$. The signal averaging device, Eclipse, plays a critical role in recording charge profiles at different phase angles. The Eclipse software on the computer could enable Eclipse to start triggering the pulse generator, and at the same time, recording data at the same frequency. Pulses applied to the PEA system can cause acoustic waves that are a representation of the space charge. The piezoelectric transducer transforms the captured acoustic signals that contain the information of charge distributions into voltage signals. The amplified voltage signals at different phase angles will then be recorded by Eclipse in series and the recorded data will be transferred back to the computer for further analysis.

\section{SIMULATION UNDER AC VOLTAGES}

\subsection{COMPARISON BETWEEN MEASUREMENT RESULTS AND SIMULATION RESULTS}

The charge evolution process in low-density polyethylene with a thickness of $100 \mu \mathrm{m}$ when applying an electric field of $50 \mathrm{~Hz} 60 \mathrm{kV} / \mathrm{mm}$ was measured for 4 hours. After subtracting capacitive charges, bulk charges and induced charges are shown in Figure 3a. The simulation results of charge profiles inside the sample during 4 hours of AC stressing are shown in Figure $3 b$.
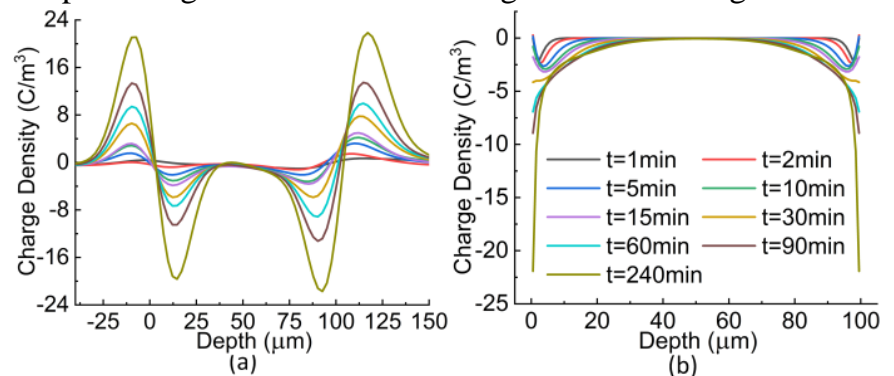

Figure 3. Charge distributions after 4 hours of AC stressing; (a) measurement results after removing the capacitive charges, (b) numerical simulation results.

From measurement results, despite the constant injection and extraction of charge carriers under AC voltage, there are negative charges accumulated near both electrodes. However, the distributions of charges near the upper and lower electrode are different. There are two reasons that caused this asymmetrical distribution. Firstly, the material of the upper electrode is semicon while that of the lower electrode is aluminum. The injection barrier for semicon is smaller than that for aluminum. Thus, the charge density near the upper electrode is a bit larger than that near the lower electrode. Secondly, due to the dispersion of acoustic signal, the charge peak near the upper electrode is a bit widened. The number of negative charges increases with stressing time and these charges move slightly deeper into the sample. After 1 hour of AC stressing, the maximum charge density near the lower electrode is 7.3 $\mathrm{C} / \mathrm{m}^{3}$. After 4 hours of AC stressing, the maximum charge density near the lower electrode reaches $19.6 \mathrm{C} / \mathrm{m}^{3}$. Negative charges are accumulated between 0-39.0 $\mu \mathrm{m}$ near the lower electrode and between 51.2-100 $\mu \mathrm{m}$ near the upper electrode.

From simulation results, the bulk charge distribution is symmetrical since the injection barriers for both electrodes are the same. Generally, the number of negative charges inside the sample becomes larger and these charges migrate deeper into the sample with time. After 1 hour, the maximum charge density near both electrodes is about $6.9 \mathrm{C} / \mathrm{m}^{3}$. After 4 hours, the maximum charge density near both electrodes is about $21.9 \mathrm{C} / \mathrm{m}^{3}$. Negative charges are accumulated between 0-39.5 $\mu \mathrm{m}$ near the lower electrode 
and between 60.5-100 $\mu \mathrm{m}$ near the upper electrode. The good agreement between simulation and experimental results verify the feasibility of parameters used for simulation.

The accumulated negative charges near both electrodes distort the electric field distribution. When the applied voltage is positive, the accumulated negative charges alleviate the field strength near the left electrode while the field strength near the right electrode is intensified.

\subsection{SIMULATION OF AC BREAKDOWN}

During a breakdown test, a ramping voltage $V$ is applied to the sample until breakdown occurs. The applied voltage $V$ in numerical simulation study is in the form of:

$V=R \times t \times \sin (2 \pi f t)$

where $R$ is the ramping rate that equals to $100 \mathrm{~V} / \mathrm{s}, t$ is time, $f$ is the frequency that equals to $50 \mathrm{~Hz}$.

During simulation, if there is any place inside the sample that the field strength reaches $500 \mathrm{kV} / \mathrm{mm}$, it is considered that breakdown happens [24]. The breakdown strength equals to the applied voltage $V$ divided by sample thickness $d$, set as $100 \mu \mathrm{m}$.

Since breakdown is determined by reaching a critical field level, a rigorous field computation is of great importance. A shorter time step and a finer meshing can produce more precise results while significantly increasing the computation time. Thus, appropriate values of time step and grid spacing should be chosen for the simulation of breakdown process. For a $100 \mu \mathrm{m}$ sample, the variation of simulated breakdown strength with time step and grid spacing is shown in Figure 4.

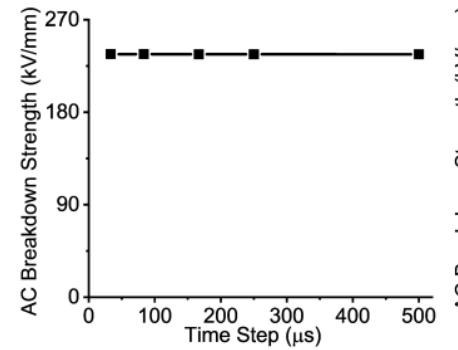

(a)

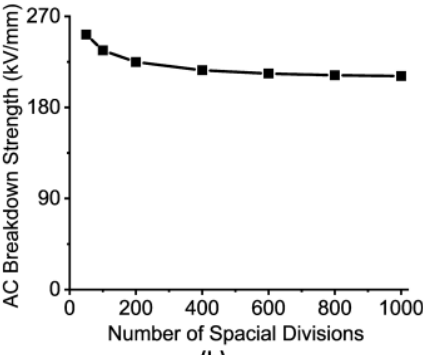

(b)
Figure 4. Variation of simulated breakdown strength; (a) time step, (b) grid spacing.

With time step varying from 50 to $500 \mu \mathrm{s}$, breakdown strength remains constant as $236.5 \mathrm{kV} / \mathrm{mm}$. The value of time step is set as $250 \mu \mathrm{s}$. With the number of space divisions increasing from 50 to 600 , the breakdown strength decreases from 236.4 to $213.5 \mathrm{kV} / \mathrm{mm}$. This is because when the applied field strength is very high, the large number of recombined charges near both electrodes cause drastic changes of parameters due to material degradation. A finer mesh will help to resolve the electrode effects. When the number of divisions further increases from 600 to 1000 , the change of simulated breakdown strength is less than $1 \%$ while the computation time is more than three times longer. Therefore, the number of divisions is set as 600 .

For a $100 \mu \mathrm{m}$ thick sample, the simulation results of charge profiles, electric field distribution at the moment of breakdown and variation of parameters with time is shown in Figure 5.

The simulated breakdown strength is $213.5 \mathrm{kV} / \mathrm{mm}$ and breakdown occurs at phase angle $81^{\circ}$. Since this phase is in the positive cycle, $x=100 \mu \mathrm{m}$ is at a positive voltage while $x=0 \mu \mathrm{m}$ is grounded. Thus, the number of positive charges near the right electrode is larger than that near the left electrode. Next to the positive charges near both electrodes, there is an obvious negative charge accumulation. The frequent injection and extraction of charges with different polarities under AC voltage leads to a larger number of recombined charges near the surface. The energy emitted by recombination creates more defects, which are deep traps in the simulation model, and interfere with the migration of charges. Since the trapping coefficient for electrons is higher than that for holes and the injection barrier for electrons is smaller, the accumulated charges inside the sample are mainly negative. When the voltage polarity switches from negative to positive, these accumulated negative charges will severely distort electric field and eventually leads to breakdown.

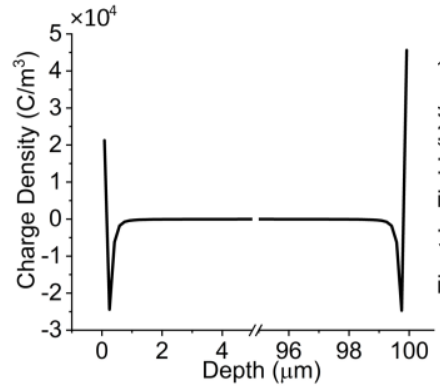

(a)

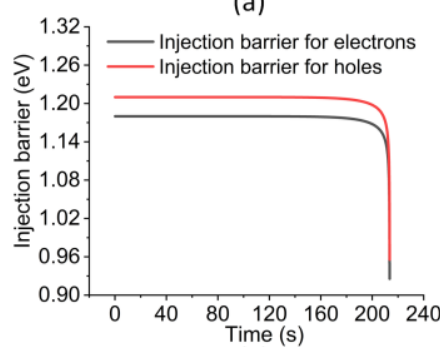

(c)

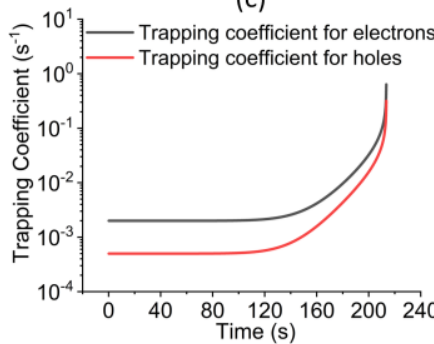

(e)

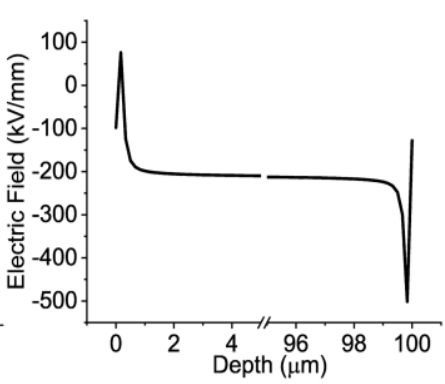

(b)

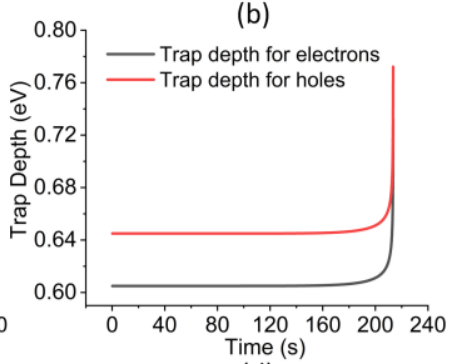

(d)

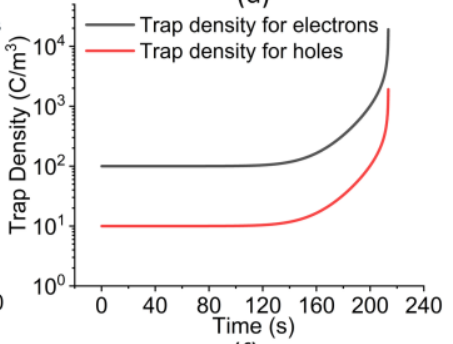

(f)
Figure 5. Simulation results of AC breakdown; (a) charge profile at breakdown, (b) electric field distribution at breakdown, (c) variation of injection barrier with time, (d) variation of trap depth with time, (e) variation of trapping coefficient with time, and (f) variation of trap density with time.

The variation of parameters with time, as shown in Figure 6, is at position $x=0.0833 \mu \mathrm{m}$, which is in the first element. The number of recombined charges at this position is the largest and the change of parameters is the largest. For electrons, the injection barrier decreases from 1.18 to $0.926 \mathrm{eV}$. The trap depth increases from 0.605 to $0.732 \mathrm{eV}$. The trapping rate increases from 0.002 to $0.638 \mathrm{~s}^{-1}$. The trap density increases from 100 to $19179 \mathrm{C} \cdot \mathrm{m}^{-3}$. Parameters change faster with time and the increasing voltage. Drastic changes occur near 210s, where the field strength is $210 \mathrm{kV} / \mathrm{mm}$, and breakdown occurs very soon.

\subsection{AC BREAKDOWN STRENGTHS WITH DIFFERENT FREQUENCIES}

By changing the frequency of the applied AC ramping voltage 
to $0.5,1,5,10$ and $50 \mathrm{~Hz}$, the simulated breakdown strengths are $224.3,216.9,214.0,213.8$ and $213.5 \mathrm{kV} / \mathrm{mm}$, respectively. The AC breakdown strength decreases with increasing frequency, which is in accordance with experimental results [25].

Since the simulated breakdown could occur either near the left electrode or the right electrode, a plot of charge profiles at the moment of breakdown might not show the influence of frequency on charge accumulation and transport clearly. Therefore, charge profiles at the same phase in the breakdown

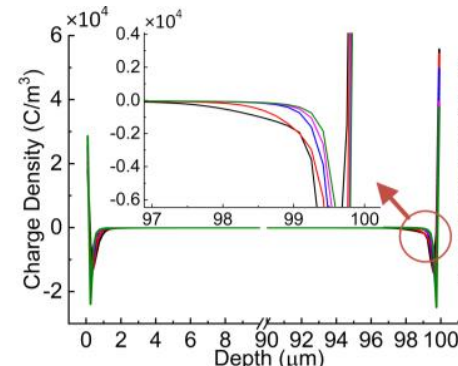

(a)

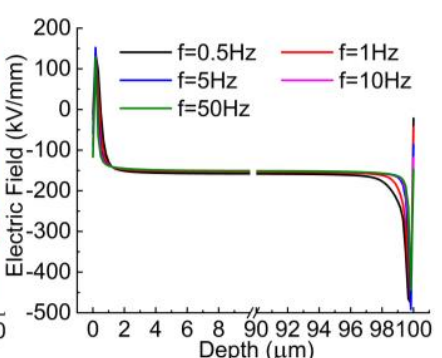

(b)
Figure 6. Simulation results at phase $45^{\circ}$ in breakdown cycle with different $f$ applied; (a) charge profiles, (b) electric field distribution.

cycle are shown in Figure 6. The selected phase angle is $45^{\circ}$.

Through comparison between the charge profiles at different frequencies, charges move deeper into the sample at lower frequencies while at higher frequencies, more charges are stuck near the surface. This is because at low frequencies, there is more time in one cycle for charges to move deeper while at high frequencies, charges are more frequently injected and extracted. The more frequent recombination near the surface generates more traps to suppress the migration of charges, which results in a larger amount of negative charges accumulated near the surface at higher frequencies.

With more negative charges accumulated near the surface, when voltage polarity switches from negative to positive, the field distortion is more serious at higher frequencies. Thus, the breakdown strength decreases with the increasing frequency.

\subsection{AC BREAKDOWN STRENGTHS WITH DIFFERENT RAMPING RATES}

When the frequency is $50 \mathrm{~Hz}$, by changing the ramping rate of the applied voltage to 50,100, 150 and $200 \mathrm{~V} / \mathrm{s}$, the AC breakdown strengths are 197.3, 213.5, 223.5 and $230.8 \mathrm{kV} / \mathrm{mm}$, respectively. The breakdown strength increases with the increasing ramping rate, which agrees well with experimental

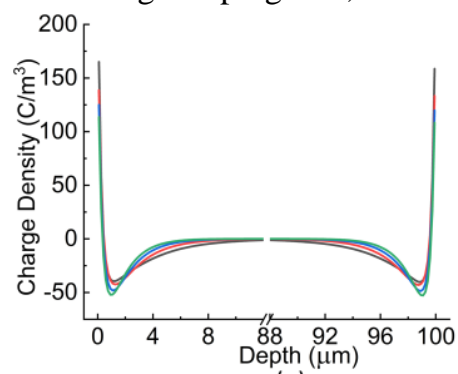

(a)

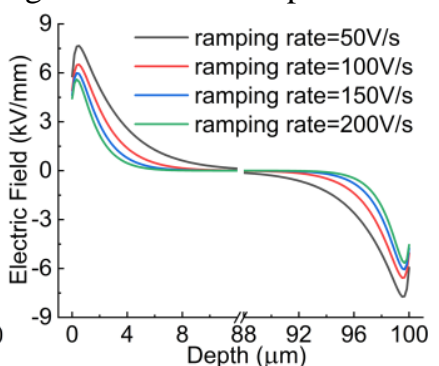

(b)
Figure 7. Simulation results at $R \times t=18 \mathrm{kV}$ with different ramping rates applied; (a) charge profiles, (b) electric field distribution.

results [25].

When $R$ multiplied by $t$ equals to $18 \mathrm{kV}$, the charge profiles and electric field distributions with different ramping rates are shown in Figure 7. The phase angle is $0^{\circ}$, which shows the influence of accumulated charges on the electric field distribution more clearly without the applied field. The influence of ramping rate is closely related to the time duration of voltage application. When $R$ multiplied by $t$ equals to $18 \mathrm{kV}$, if the ramping rate is $50 \mathrm{~V} / \mathrm{s}$, the time length of voltage application is 360s. Yet for the voltage with a ramping rate of $100 \mathrm{~V} / \mathrm{s}$, the time is only $180 \mathrm{~s}$. This shorter time will result in a smaller amount of injected charges. And it is more difficult for charges to move deeper into the sample with a shorter time. With the increasing ramping rate, although the negative charge density near both electrodes is larger, the total amount of accumulated negative charges inside the sample is smaller, which would result in a less-distorted electric field, as shown in Figure $7 \mathrm{~b}$. Therefore, the breakdown strength increases with increasing ramping rate.

\section{SIMULATION UNDER AC-DC COMBINED VOLTAGES}

After understanding the breakdown process under $\mathrm{AC}$ voltages and how the parameters of AC voltage influences the charge transport characteristics, we move a step further towards the simulation of AC-DC combined breakdown strengths of polyethylene based on the modified bipolar charge transport model. Through simulation, how AC component and DC component affect the migration and accumulation of charges

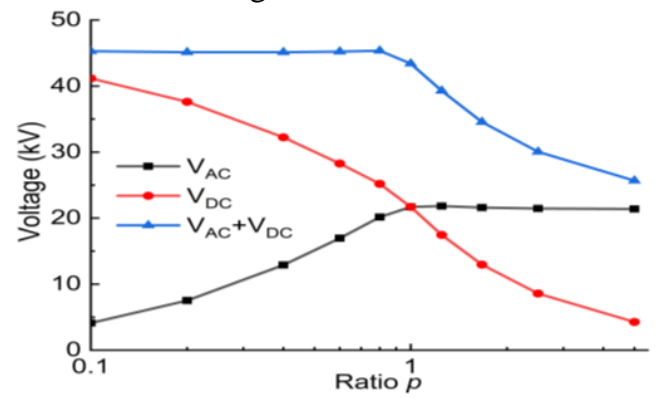

Figure 8. Simulation breakdown voltages of polyethylene versus $p$.

during the evolution of breakdown can be understood.

If the applied AC-DC combined voltage is in the form:

$V=V_{\mathrm{DC}}+V_{\mathrm{AC}} \times \sin (2 \pi f t)=R \times t+p \times R \times t \times \sin (2 \pi f t)$

where $V_{\mathrm{DC}}$ is the value of the DC component, $V_{\mathrm{AC}}$ is the peak value of the AC component, $R$ is set as $100 \mathrm{~V} / \mathrm{s}, p$ is the ratio of $\mathrm{AC}$ to DC component. When $p$ changes, the actual ramping rate of AC component changes. For instance, if $p$ equals to 10 , the actual ramping rate of $\mathrm{AC}$ component is $1000 \mathrm{~V} / \mathrm{s}$, which will significantly increase the breakdown strength. Therefore, the influence of ramping rate on breakdown strength should be excluded as much as possible to mainly focus on analyzing the influence of $p$ on charge accumulation characteristics and ACDC combined breakdown strengths. For $p<1$, when DC component is dominant, the ramping rate of $\mathrm{DC}$ component is kept constant as $100 \mathrm{~V} / \mathrm{s}$. For $p>1$, when $\mathrm{AC}$ component is dominant, the ramping rate of $\mathrm{AC}$ component is kept constant as $100 \mathrm{~V} / \mathrm{s}$. The applied voltage is in the form:

$V=\left\{\begin{array}{l}R \times t+p \times R \times t \times \sin (2 \pi f t), p<1 \\ \frac{1}{p} \times R \times t+R \times t \times \sin (2 \pi f t), p>1\end{array}\right.$

For different ratios of $p$, when breakdown happens, the 
simulated values of $\mathrm{AC}$ component, DC component and the breakdown voltage are shown in Figure 8.

From the simulation results, with the increasing ratio $p$, the breakdown voltage, which is the sum of AC component and DC component, firstly shows a slight increase followed by an obvious decrease. The DC component shows a monotonic decrease with the increasing ratio $p$ while the $\mathrm{AC}$ component firstly increases and then almost remains constant. This trend is in good agreement with the results from experiments $[3,4]$. The charges profiles and field distributions when $p<1$ and $p>1$ are analyzed separately in the following parts.

\subsection{CHARGE AND FIELD DISTRIBUTION FOR $\mathbf{P}<\mathbf{1}$}

When $p$ equals to $0.2,0.4,0.6$ and 0.8 , the summed-up breakdown voltages are $45.14,45.14,45.23$ and $45.36 \mathrm{kV}$, respectively. The amplitude of $\mathrm{AC}$ component increases while that of DC component decreases.

When $p<1$, the polarity of the applied voltage stays positive. Homo charges are injected from both electrodes, which alleviate the electric field near the surface yet intensify the electric field in the middle part of the sample. Therefore, when $p<1$, breakdown occurs in the bulk instead of near the surface.

When the time duration of voltage application is the same, the value of DC component is the same. The amplitude of $\mathrm{AC}$ component increases with the increasing ratio $p$. The higher amplitude of AC component has two major influences. Firstly, when the phase angle is between $0^{\circ}$ and $180^{\circ}$, the applied voltage is higher. According to equation (1), a higher electric field strength results in a larger injection current, which means more homo charges are injected into the sample. According to equation (3), a higher electric field also means a larger mobility. Charges can move deeper into the sample with a higher migration speed. Secondly, when the phase angle is between $180^{\circ}$ and $360^{\circ}$, the applied voltage is lower. The smaller electric field results in a smaller number of injected charges and a smaller mobility. The charge distribution is a combined result of these two influences.

The simulation results at the same time $(252.025 \mathrm{~s})$ with

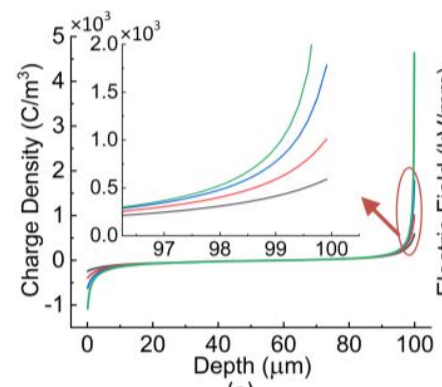

(a)

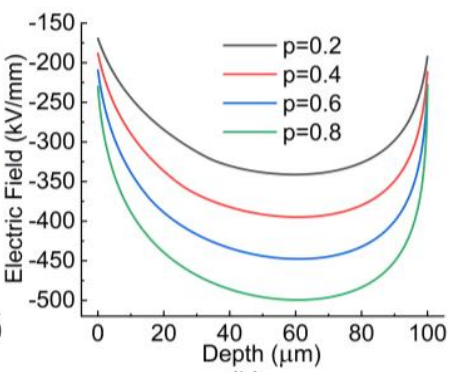

(b)
Figure 9. Simulation results at $\mathrm{t}=252.025 \mathrm{~s}$ with different ratio $p$ : (a) charge profiles (b) electric field distribution.

different ratio $p$ are plotted in Figure 9. 252.025s is the time breakdown happens when ratio $p$ equals to 0.8 . Through comparison, the first influence plays the dominant role. With a higher ratio $p$, the increasing $\mathrm{AC}$ component introduces a larger number of injected charges into the sample. The higher the maximum voltage amplitude $\left(V_{\mathrm{AC}}+V_{\mathrm{DC}}\right)$ is, the larger number of homo charges are injected.

For breakdown in the bulk of sample to happen, a specific amount of homo charges needs to be injected to cause enough electric field distortion. Under AC-DC combined voltages, these injected charges can be considered as two parts, the ones from DC component and the ones from AC component. Since a larger $\mathrm{AC}$ component leads to a larger amount of injected charges, the corresponding DC component decreases with the increasing AC ratio, as shown in the left part of Figure 8.

When the applied voltage is the same, the more homo charges are accumulated inside the sample, the lower the breakdown strength is. Therefore, to analyze the relationship between breakdown voltage and ratio $p$, the simulation results for an applied voltage of $V_{\mathrm{AC}}+V_{\mathrm{DC}}=45 \mathrm{kV}$ are shown in Figure 10 .

In Figure 10, the amount of injected homo charges decreases

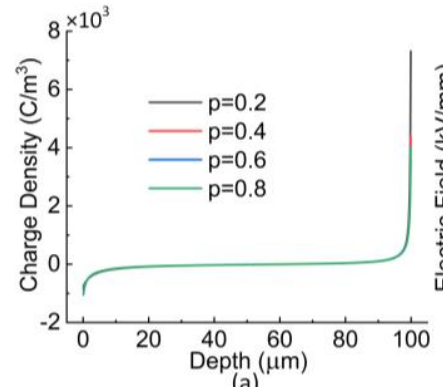

(a)

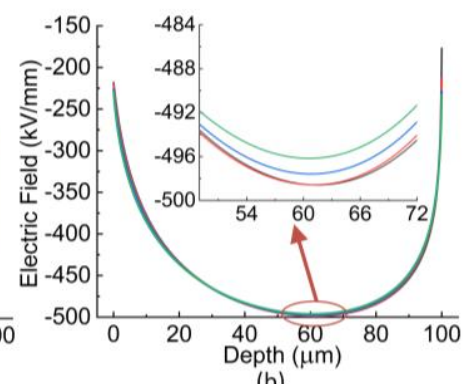

(b)
Figure 10. The simulation results when $\mathrm{V}_{\mathrm{AC}}+\mathrm{V}_{\mathrm{DC}}=45 \mathrm{kV}$ with different $\mathrm{AC}$ ratio $p$ : (a) charge profiles (b) electric field distribution.

with increasing ratio $p$. This is because of the different time duration of voltage application. Since the ramping rate of DC component is kept constant, with $V_{\mathrm{AC}}+V_{\mathrm{DC}}$ being the same, the smaller $p$ is, the longer the voltage is applied. A longer voltage application means more charges are injected into the sample, which result in a larger electric field distortion, as shown in Figure 11b. Thus, the breakdown voltage increases slightly with the increasing ratio $p$.

\subsection{CHARGE AND FIELD DISTRIBUTION FOR P>1}

When $p$ equals to $1.25,1.67,2.5$ and 5 , the breakdown voltages are $39.29,34.56,30.05$ and $25.67 \mathrm{kV}$, respectively. With the increasing ratio $p$, the amplitude of $\mathrm{AC}$ component almost remains constant while that of DC component decreases.

When $p>1$, the AC component becomes dominant. The applied voltage changes polarity in each cycle. Thus, the recombination between positive and negative charges plays an important role in breakdown. The energy emitted through recombination leads to the generation of physical and chemical defects. Since the overall trap depth increases with material degradation [21], the increasing number of deep traps is considered as the major influence. With a larger trap density and trapping rate, the increasing number of charges accumulated near the electrodes distorts the electric field and eventually leads to breakdown.

Breakdown always occurs near the left electrode and near phase angle $270^{\circ}$. The reason is as follows. From equation (10), for $p>1$, the time duration that the applied voltage is positive is longer than the time duration that the applied voltage is negative. Thus, near the left electrode, more negative charges are injected while near the right electrode, more positive charges are injected. Since the injection barrier for electrons is smaller and the mobility for electrons is higher, more negative charges are injected into the sample than positive charges. Furthermore, negative charges migrate much deeper into the sample, which causes a more severe field distortion near the left electrode than near the right electrode. Therefore, when the 
voltage polarity switches from positive to negative, especially approaching phase angle $270^{\circ}$, the intensified field strength near the left electrode is larger than that near the right electrode.

With the same voltage $V_{\mathrm{AC}}+V_{\mathrm{DC}}$ applied, the higher the field strength is near the left electrode at phase angle $270^{\circ}$, the lower the breakdown voltage will be. Therefore, the simulation results when $V_{\mathrm{AC}}+V_{\mathrm{DC}}=25.2 \mathrm{kV}$ with different ratio $p$ are shown in Figure 11.

With the same voltage, a higher ratio $p$ means a smaller DC component and a larger AC component. With a smaller DC

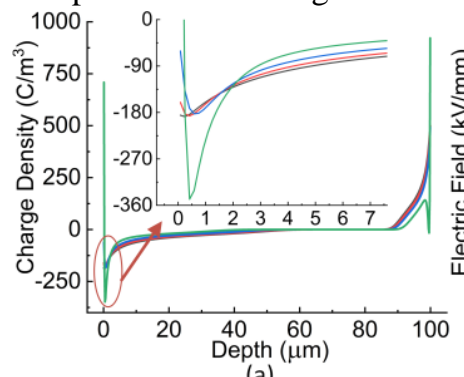

(a)

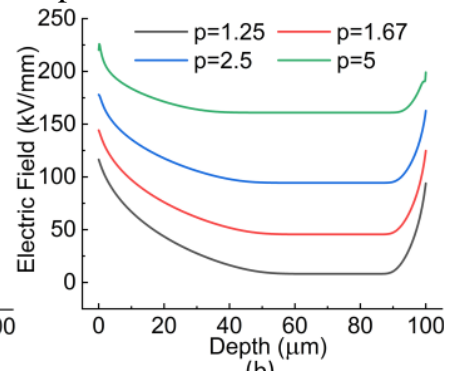

(b)
Figure 11. The simulation results when $\mathrm{V}_{\mathrm{AC}}+\mathrm{V}_{\mathrm{DC}}=25.2 \mathrm{kV}$ with different $\mathrm{AC}$ ratio $p$ : (a) charge profiles (b) electric field distribution.

component, charges are more easily stuck near the surface instead of migrating into the sample, as shown in Figure 11a. With a larger $\mathrm{AC}$ component, the absolute value of maximum negative voltage, which equals to $V_{\mathrm{AC}}-V_{\mathrm{DC}}$, increases with the increasing ratio $p$. Therefore, when the voltage polarity switches from positive to negative, the applied voltage and electric field strength is larger at higher ratios, as shown in Figure 11b. Therefore, the breakdown voltage decreases with the increasing ratio $p$.

\subsection{COMPARISON WITH EXPERIMENTAL MODEL}

Based on a large amount of experimental results, researchers have proposed a mathematical model describing the relationship between AC-DC combined breakdown voltage and ratio $p$, as follows [3]:

$$
\begin{aligned}
& V_{D C}=\frac{2}{\pi} \times A \times \operatorname{arccot} \frac{p}{\alpha} \\
& V_{A C}=\frac{2}{\pi} \times B \times \arctan \frac{p}{\beta}
\end{aligned}
$$

where $A$ is the DC breakdown voltage, $B$ is the AC breakdown voltage, $\alpha$ equals to $p$ when the DC component equals to $0.5 A$, $\beta$ equals to $p$ when the AC component equals to $0.5 B$.

The simulation results are compared with the results obtained from this model which is based on experimental results to see whether the variation trend shows a good agreement. From simulation results, for samples with a thickness of $100 \mu \mathrm{m}$, the AC breakdown voltage and DC breakdown voltage are 21.4 and $45.5 \mathrm{kV}$, respectively. To get the values of $\alpha$ and $\beta$, cubic spline interpolation is used to fit the curves. When DC component equals to $22.7 \mathrm{kV}$, ratio $p$ equals to 0.93 . When $\mathrm{AC}$ component equals to $10.7 \mathrm{kV}$, ratio $p$ equals to 0.32 . The simulation results and calculation results from the model are shown in Figure 12.

The results calculated from the model based on experimental results agree well with the simulation results. With the increasing ratio $p$, the AC-DC combined voltage firstly shows a slight increase followed by a drastic decrease. The only difference is that the decrease occurs at a larger $p$ in simulation results than those in the calculated results from the model.

This difference could have several possible reasons. Firstly, for the breakdown tests, the experimental results are a bit scattered. Thus, the obtained breakdown strengths from

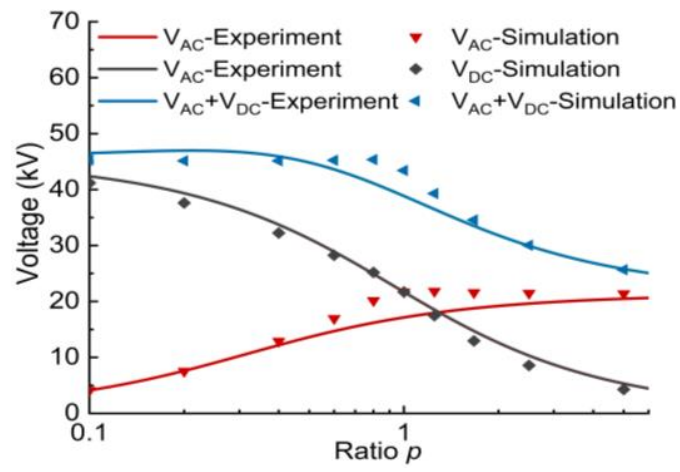

Figure 12. Comparison between simulation results and calculation results from proposed model on AC-DC combined breakdown voltage.

experiments might not be very precise. Secondly, a simplified simulation model of breakdown might be another reason. In this paper, breakdown happens when there is a certain position inside the sample that the electric field strength reaches 500 $\mathrm{kV} / \mathrm{mm}$. However, based on previous researches, breakdown is rather complex and defined by a number of processes: charge injection, material modification by carrier recombination, material damage caused by impact excitation, formation of a tubular cavity and the formation of a complete conducting filamentary path. The simulation model in this paper mainly focuses on the influence of injected and trapped charges on material degradation and electric field distortion. The latter two processes are not considered as the focus in the simulation model adopted in this paper.

\section{CONCLUSIONS}

In this paper, the influence of the $\mathrm{AC}$ voltage frequency, ramping rate and the ratio of $\mathrm{AC}$ component to $\mathrm{DC}$ component in composite voltages on the accumulation of charges and breakdown strengths are studied through numerical simulation. The conclusions are as follows.

(1) The more frequent charge recombination at higher frequencies results in a higher trap density and a larger trapping coefficient. More negative charges are accumulated near the surface, which leads to a lower breakdown strength.

(2) With increasing voltage ramping rate, the breakdown strength increases due to a shorter voltage application time, which limits the amount of injected charges.

(3) The AC-DC combined breakdown voltage firstly shows a slight increase followed by a drastic decrease with increasing AC-DC ratio. The simulation results agree well with the variation trend observed in previous experiments.

Local thermal instability also plays an important role in the breakdown process during ramping voltage tests. The increase of temperature will lead to a more obvious charge injection, a higher mobility and a larger de-trapping rate. The inclusion of thermal instability will be the focus of next part of work. 


\section{ACKNOWLEDGEMENTS}

The authors want to express their sincere gratitude towards the colleagues at Tony Davies High Voltage Lab for their support. S. Zhang thanks the China Scholarship Council (CSC) for providing the scholarship to become a visiting Ph.D. student at the University of Southampton (No. 201603780058).

\section{REFERENCES}

[1] Y. Wang, X. Wei, Q. Chen, Y. Huang, and H. Nie, "Breakdown characteristics of converter transformer insulation under composite AC and DC voltage," $9^{\text {th }}$ Int. Conf. Prop. Applica. Dielectr. Materi. (ICPADM), 2009, pp. 634-637.

[2] Y. Wang, J. Li, Y. Wang, and S. Grzybowski, "Electrical breakdown properties of oil-paper insulation under AC-DC combined voltages," IEEE Int. Power Modu. High Volt. Conf. (IPMHVC), 2010, pp. 115-118.

[3] S. Grzybowski and R. L. Mcmellon, "Electrical breakdown strength of XLPE cables under combined AC-DC voltage," IEEE Southeastcon 95 Visualize the Future, 1995, pp. 63-66.

[4] S. Grzybowski and J. Fan, "Electrical breakdown strength of $5 \mathrm{kV}$ imperfect XLPE cable after combined AC-DC excitation," Аnnи. Rep. Conf. Electr. Insul. Dielect. Phenom.(CEIDP), 1996, pp. 295-298.

[5] Y. Zhang, J. Lewiner, C. Alquie and N. Hampton, "Evidence of strong correlation between space-charge buildup and breakdown in cable insulation,” IEEE Trans. Dielectr. Electr. Insul., vol. 3, no. 6, pp. 778-783, 1996.

[6] L. A. Dissado, G. Mazzanti, and G. C. Montanari, "The role of trapped space charges in the electrical aging of insulating materials," IEEE Trans. Dielectr. Electr. Insul., vol. 4, no.5, pp. 496-506,1977.

[7] C. Zhou and G. Chen, "Space charge and AC electrical breakdown strength in polyethylene," IEEE Trans. Dielectr. Electr. Insul., vol. 24, no.1, pp. 559-566, 2017.

[8] S. L. Roy, G. Teyssedre, C. Laurent, G. C. Montanari, and F. Palmieri, "Description of charge transport in polyethylene using a fluid model with a constant mobility: fitting model and experiments," J. Phys. D: Appl. Phys., vol. 37, no. 7, pp. 1427-1436, 2006.

[9] M. Meunier, N. Quirke and A. Aslanides, "Molecular modeling of electron traps in polymer insulators: Chemical defects and impurities," J. Chem. Phys., vol. 115, no. 6, pp. 2876-2881, 2001.

[10] K. C. Kao, "New theory of electrical discharge and breakdown in low mobility condensed insulators," J. Phys. D: Appl. Phys., vol. 55, no.3, pp. $752-755,1983$

[11] C. Laurent, G. Teyssedre, S. L. Roy, and F. Baudoin, "Charge dynamics and its energetic features in polymeric materials," IEEE Trans. Dielectr. Electr. Insul., vol.20, no.2, pp. 357-381, 2013.

[12] M. Meunier and N. Quirke, "Molecular modeling of electron trapping in polymer insulators," J. Chem. Phys., vol.113, no.1, pp. 369-376, 2000.

[13] M. Kuik, L. J. A. Koster, G. A. H. Wetzelaer, and P. W. M. Blom, "TrapAssisted Recombination in Disordered Organic Semiconductors," Phys. Rev. Lett., vol. 107, no. 25, pp. 256805(5pp), 2011.

[14] M. Kuik, L. J. A. Koster, A. G. Dijkstra, G. A. H. Wetzelaer, and P. W. M. Blom, "Non-Radiative Recombination Losses in Polymer LightEmitting Diodes," Org. Electron., vol. 13, no. 6, pp. 969-974, 2012.

[15] S. L. Roy, G. Teyssedre, and C. Laurent, "Numerical methods in the simulation of charge transport in solid dielectrics," IEEE Trans. Dielectr. Electr. Insul., vol. 13, no.2, pp. 239-246, 2006.

[16] B. P. Leonard, "Universal limiter for transient interpolation modeling of the advective transport equations: the ULTIMATE conservative difference scheme," NASA Techn. Memor., vol. 100916, pp. ICOMP-8811,1988

[17] K. Wu and L. A. Dissado, "Model for electrical tree initiation in epoxy resin," IEEE Trans. Dielectr. Electr. Insul., vol. 12, no.4, pp. 655-668, 2005

[18] C. Zhou, "Space charge dynamics in polyethylene under periodical high voltage electric fields", PhD dissertation, Dept. of Electr. and Compu. Sci., University of Southampton, United Kingdom, 2017.

[19] G. Blaise, "Charge localization and transport in disordered dielectric materials," J. Electrostat., vol.50, no.2, pp. 69-89, 2001.

[20] G. Teyssèdre and C. Laurent, "Charge transport modeling in insulating polymers: from molecular to macroscopic Scale," IEEE Trans. Dielectr. Electr. Insul., vol.12, no.5, pp. 857-875, 2005.
[21] N. Liu, M. He, H. Alghamdi, G. Chen, M. Fu, R. Li, and S. Hou, "An improved model to estimate trapping parameters in polymeric materials and its application on normal and aged low-density polyethylenes," J. Phys. D: Appl. Phys., vol. 118, no. 6, pp. 1147-1158, 2015.

[22] G. Mazzanti, G. C. Montanari, and J. M. Alison, "A space-charge based method for the estimation of apparent mobility and trap depth as markers for insulation degradation-theoretical basis and experimental validation," IEEE Trans. Dielectr. Electr. Insul., vol.8, no.2, pp. 187-197, 2003.

[23] C. Zhou and G. Chen, "Space charge behaviours in polyethylene under combined AC and DC electric fields," IEEE Annu. Rep. Conf. Electr. Insul. Dielect. Phenom.(CEIDP), 2014, pp. 848-851.

[24] K. Matsui, Y. Tanaka, T. Takada and T. Fukao, "Space charge behavior in low density polyethylene at pre-breakdown," IEEE Trans. Dielectr. Electr. Insul., vol. 12, no. 3, pp. 406-415, 2005.

[25] M. Nagao, K. Takano, Y. Mizuno and M. Kosaki, "Intrinsic AC Breakdown of Low-Density Polyethylene Film above Room Temperature," $3^{\text {rd }}$ Int. Conf. Prop. and Appl. Dielectr. Mat.(ICPADM), 1991, pp. 1165-1168.

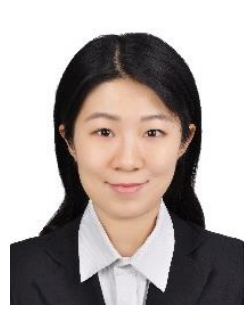

Siyu Zhang was born in Hebei province in China in 1993. In 2014, she received the bachelor's degree in electrical engineering from Xi'an Jiaotong University. Now she is working as a Ph.D. candidate majoring in high voltage and insulation technology at Xi'an Jiaotong University. Her area of interest lies in the space charge measurement technique and numerical simulation of charge transport behaviors. She has been awarded a scholarship from China Scholarship Council and is now working as a vising Ph.D student at the University of Southampton.

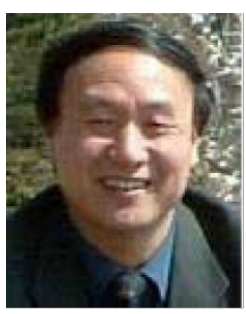

Zongren Peng was born in Shaanxi province, China, in 1953. In 1977, he graduated from Xi'an Jiaotong University, China, majoring in electrical engineering. He was a visiting researcher at Musashi Institute of Technology, Japan and University of Southampton, UK. Currently, he is a professor at the school of electrical engineering in Xi'an Jiaotong University. $\mathrm{He}$ is also a leading expert of the state grid in China. His researches include the design and optimization of insulation structures inside power equipment, the dielectric properties and space charge characteristics of insulation materials.

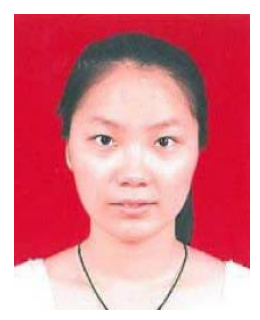

Churui Zhou was born in China in Hubei province in 1991. In 2013, she received the B.Eng. degree in electrical engineering from Huazhong University of Science and technology, China. In the same year, she received the B.Eng. degree in electrical from University of Birmingham, UK. Since then, she has been working as a Ph.D. student in the University of Southampton, UK. Her main research interest lies in experimental and numerical modelling of space charge dynamics in polyethylene.

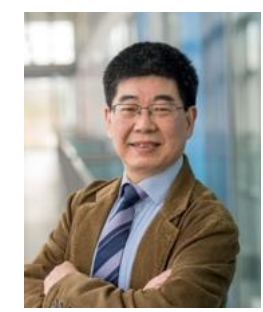

George Chen was born in China in 1961. He received the BEng (1983) and MSc (1986) degrees in electrical engineering from Xi'an Jiaotong University, China. After he obtained the PhD degree (1990) from the University of Strathclyde, UK, on the work of permanent changes in electrical properties of irradiated low-density polyethylene, he joined the University of Southampton as a postdoctoral research fellow and became a senior research fellow subsequently. In 1997 he was appointed as a research lecturer and promoted to a reader in 2002. He is now professor of $\mathrm{HV}$ engineering at the University of Southampton and a visiting professor of Xi'an Jiaotong University. Over the years, he has developed a wide range of interests in $\mathrm{HV}$ engineering and electrical properties of materials and has published more than 300 papers. 